\title{
Personal Clipboards for Individual Copy-and-Paste on Shared Multi-User Surfaces
}

\author{
Dominik Schmidt \\ Hasso Plattner Institute \\ Potsdam, Germany \\ dominik.schmidt@hpi.uni-potsdam.de
}

\author{
Corina Sas, Hans Gellersen \\ Lancaster University \\ Lancaster, UK \\ \{corina,hwg\}@comp.lancs.ac.uk
}

\begin{abstract}
Clipboards are omnipresent on today's personal computing platforms. They provide copy-and-paste functionalities that let users easily reorganize information and quickly transfer data across applications. In this work, we introduce personal clipboards to multi-user surfaces. Personal clipboards enable individual and independent copy-and-paste operations, in the presence of multiple users concurrently sharing the same direct-touch interface. As common surface computing platforms do not distinguish touch input of different users, we have developed clipboards that leverage complementary personalization strategies. Specifically, we have built a context menu clipboard based on implicit user identification of every touch, a clipboard based on personal subareas dynamically placed on the surface, and a handheld clipboard based on integration of personal devices for surface interaction. In a user study, we demonstrate the effectiveness of personal clipboards for shared surfaces, and show that different personalization strategies enable clipboards, albeit with different impacts on interaction characteristics.
\end{abstract}

\section{Author Keywords}

Multi-touch surfaces; clipboards; copy-and-paste.

\section{ACM Classification Keywords}

H.5.2. Information Interfaces and Presentation (e.g. HCI): User Interfaces-Input devices and strategies, Interaction styles.

\section{General Terms \\ Human Factors; Design.}

\section{INTRODUCTION}

Today's desktop and mobile computing platforms are hardly imaginable without clipboards and the associated copy-andpaste functionalities. Clipboards facilitate workflows by allowing users to swiftly rearrange, duplicate, or temporarily store information. They also provide a standard mechanism for data transfer across different applications. Conceptually, they implement a background buffer that is easily accessible through basic copy-and-paste operations from any application.

Permission to make digital or hard copies of all or part of this work for personal or classroom use is granted without fee provided that copies are not made or distributed for profit or commercial advantage and that copies bear this notice and the full citation on the first page. To copy otherwise, or republish, to post on servers or to redistribute to lists, requires prior specific permission and/or a fee.

CHI 2013, April 27-May 2, 2013, Paris, France.

Copyright 2013 ACM 978-1-4503-1899-0/13/04...\$15.00.

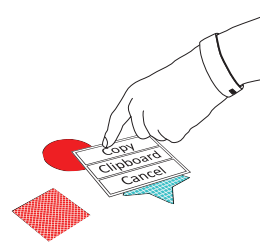

(a) Context menu

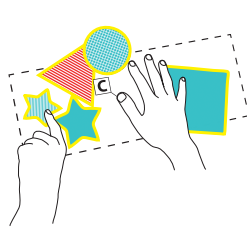

(b) Subarea

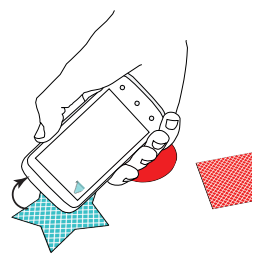

(c) Handheld
Figure 1. Three personal clipboard systems for shared surfaces, based on different personalization strategies: (a) Context menu clipboards are based on implicitly associating finger touches to users. (b) Subarea clipboards dynamically assign surface regions to users. (c) Handheld clipboards integrate mobile devices for complementary interaction.

This is a simple model and therefore easy to use with minimal mental effort [28].

Originally part of text-based editors, clipboards were adopted successfully to graphical user interfaces (GUI), have become standard in desktop applications, and are now also common on mobile devices with multi-touch interfaces, such as smart phones and tablet computers [32]. In spite of their evident utility, however, clipboards have not yet been extended for multiuser surface computing. The problem on shared surfaces is that copy-and-paste actions of users would be interleaved and confusing, as users interact simultaneously through the same medium. To realize the familiar copy-and-paste semantics on shared surfaces, users require their own clipboard, and applications must be able to distinguish input from different users to unambiguously resolve individual copy-and-paste operations.

Mainstream multi-touch surfaces do not distinguish touch from different users. To realize personal clipboards, we therefore build on complementary methods for associating touch input with individual users. Three common strategies are (a) to use additional sensing techniques for association of finger touches with users, (b) to dynamically associate surface regions with individuals, and (c) to use personal devices in conjunction with shared surfaces. We present a clipboard design and implementation for each of these strategies, with the dual aim of demonstrating alternative system solutions, and gaining insight into the implications of different personalization strategies

Figure 1 shows the three clipboard systems we have developed: (a) a context menu clipboard based on implicit user identification, (b) a clipboard coupled to a personal subarea, and (c) a handheld clipboard on a mobile phone used in stylus-like fashion for direct interaction on the surface. Each clipboard has been designed and implemented on top of existing meth- 
ods for user identification. The context menu clipboard was realized with IdWristbands [12], the subarea clipboard with HandsDown [22], and the handheld clipboard with PhoneTouch [21].

\section{Benefits of Personal Clipboards}

Conceptually, personal clipboards provide individual spaces that are exclusive to their user, within the larger shared workspace. This enables users to copy and paste items independently without interference. Moreover, users can interleave individual tasks and group tasks. Like traditional clipboards, personal clipboards reside in the background without permanently occupying surface space, but are directly accessible in the flow of touch interaction to select items to be copied, or locations for pasting.

Personal clipboards provide the following unique advantages: First, source and target location do not need to be simultaneously reachable (e.g., the target location may be at the other end of a large surface) or visible at the same time (e.g., an application switch may occur). Therefore, copy-and-pasteunlike drag-and-drop-enables users to pick up information for later pasting at different locations. Secondly, personal clipboards reduce clutter as clipboards and the enclosed items do not occupy permanent surface space. Thirdly, personal clipboards provide an exclusive space for their owners, allowing them to collect, organize, or sort copied items individually.

For example, when collaboratively creating a presentation, users may organize their workflow into successive phases. After individually searching and copying items from the web (e.g., text snippets or photos), they switch applications and closely collaborate in assembling a presentation by pasting and arranging items from their individual clipboards in the shared space.

\section{Contribution}

In this paper, we introduce personal multi-item clipboards that allow for individual copy-and-paste on shared multi-user surfaces, and study how users interact with these clipboards. The contribution of this work is three-fold: First, we present design and implementation of three personal clipboard systems, each based on a different personalization strategy. Secondly, we demonstrate that personal clipboards allow for directly carrying over familiar copy-and-paste semantics to shared surfaces, while preserving the unique advantages of traditional clipboards. Thirdly, our study also provides a direct comparison of distinct personalization strategies for surface computing. We show that all studied strategies facilitate the effective use of personal clipboards, but impact surface interaction differently.

\section{RELATED WORK}

Clipboards have evolved from buffers in early online editors designed for reuse of text "snippets" [5], and the associated notions of copy, cut, and paste have developed into universal commands in GUI and modern operating systems [28, 32]. The concept has since been extended, for example to copy and paste objects across devices [14], adapt objects to the pastecontext [30], ease copying across overlapping windows [4], or support particular practices such as programming [33]. A common extension are multi-item clipboards that let users select items to paste, for instance through a GUI in Microsoft Office, a keyboard extension [2], or a tangible interface [3]. Clipboards are also available for collaborative contexts, for example data transfer from personal devices to shared displays [15], or sharing of clips on the web [7]. In contrast to those works, we aim to extend clipboards to surface computing platforms. Our aim is to provide individual users each with their own clipboard so that they can use copy-and-paste without interference, while interacting simultaneously on the same shared surface.

Only limited research has touched on copying and pasting on shared multi-touch surfaces. DocuBits enabled copying of screen regions based on a paper-cutting metaphor, for transfer to connected personal devices but without clipboard support [8]. Wu et al. demonstrated a "cut/copy-n-paste" gesture that lets users drag and simultaneously resize objects on the surface $[35,27]$. The gesture can be used for multiple users to create their own work copy of an object, but is limited to contexts where source and target are visible at the same time and reachable in one motion. In contrast, we aim to support the richer copy-and-paste practices that users know from single-user platforms. Studies on those platforms have indicated that users copy and paste more often across windows and applications than within, and that complex patterns (copy to distributed targets, or paste composition from distributed sources) are more common than isolated copy-and-paste transactions [29].

Personal clipboards provide users with workspaces that are complementary to the surface space. In other work concerned with organization of items, storage bins support stacking of items to create groups that take less space on the surface [25]. Table trays are dynamically created around groups of items which then can be cut or copied onto the tray, moved, and pasted elsewhere on the surface [16]. Tangible drawers are virtual spaces "under the surface" that can be accessed or hidden on demand, in order to move items on or off the main workspace [9]. Personal clipboards are orthogonal to these concepts, as they provide an exclusive space to the individual users around a shared surface. They contrast storage bins and table trays as they store items in the background, and they contrast tangible drawers as they are accessed in the flow of touch interaction on the surface.

It is essential for personal clipboards that copy-and-paste actions on a shared surface can be associated with individual users, but state of the art multi-touch surfaces do not readily distinguish touch from different users. In a variety of systems, additional sensing is used to associate every touch implicitly with a user. In DiamondTouch, this was achieved with a wired connection of user and surface [6], while IR Ring [20] and IdWristbands [12] provide untethered identification based on transmitters worn by the user. Other work has used extrinsic sensing, for example Kinect to first authenticate users and then track their hands [19], or cameras under the table to identify and track users by the shoes they wear [18]. A different strategy to facilitate user identified input is to associate surface regions with users. HandsDown lets users claim surface ar- 
eas based on hand contour analysis [22], and can be used to dynamically create personal subareas on a shared space [23]. Another strategy is to use user-associated devices for input. PhoneTouch lets users provide direct touch input with their phones in a stylus-like fashion [21,24], while other work has integrated mobile phones for authentication only [10, 19]. For our purposes, we build on three of these existing approaches (i.e., IdWristbands, HandsDown, and PhoneTouch) to pursue design alternatives for clipboards that leverage different personalisation strategies.

There is a range of further work on copy-and-paste for data transfer across devices, such as Pick-and-Drop [17]. However, we focus on enabling individualized copy-and-paste on shared surfaces, and we consider data transfer across devices only in as far as we integrate personal devices for direct copy action on the surface in one of our three clipboard systems.

\section{PERSONAL CLIPBOARDS}

Personal clipboards are designed as an extension to the shared workspace of multi-touch surfaces. The general design is that each user has their own clipboard, onto which they can copy multiple items from the shared workspace. The clipboards provide background storage and users can collect items onto them that remain in the background until they are pasted back onto the main workspace. For pasting, users can select among any of the stored items and insert them directly at a chosen location on the shared workspace.

Personal clipboards provide users with the copy-and-paste functionality they know from work on single-user platforms. Multiple users can each reuse, rearrange, or transfer items in the process of working together, doing so without interference. They can organize their workflows in different ways, for example into successive phases of collecting items and using items from their clipboards, and interweave individual and collaborative tasks.

A key concern is to integrate personal clipboards into surface computing practice in a manner that lets them "stay in the flow" [1]. Copy-and-paste must be directly accessible, in the context of items they wish to copy, or locations at which they aim to paste. Access must be seamlessly integrated with an individual user's flow of multi-touch interaction, and it must not impede the interactions or disrupt the workflow of others working concurrently on the surface. At the same time, single copy-and-paste interactions must be attributable to individual users. This requires techniques that immediately identify users while they interact with a surface.

We present three systems that each demonstrate personal clipboards based on a different strategy for personalization on shared surfaces. For each system, we chose a user identification technique that implements the particular personalization strategy.

\section{Context Menu Clipboards}

Context menu clipboards build on an implicit personalization strategy, which immediately associates any finger touch to a user. Therefore, users do not need to change familiar multitouch interaction styles for identified input. We implemented these clipboards using IdWristbands as the underlying identification technique [12]. Users wear IdWristbands like common wristbands (at the hand used for surface interaction). Based on textile sports wristbands, IdWristbands do not impede hand movement. Each IdWristband continuously emits a unique identification code using infrared LED. The shared surface detects and decodes the resulting infrared light flashes. Based on proximity, it then associates finger touches to wristbands and, in turn, users.

Context menus provide immediate access to copy-and-paste anywhere on the surface, without occupying permanent screen space. We used the established Windows 7 press-and-tap gesture to invoke context menus (i.e., "Press the item with one finger, then quickly tap with another finger, while continuing to press the item with the first finger." [13]). As IdWristbands implicitly associates finger touches to corresponding users, attributing individual copy-and-paste interactions to personal clipboards is straightforward.

Figure 2 illustrates copying and pasting with context menu clipboards. To copy an item to the personal clipboard, users perform the press-and-tap gesture on top of the item and select "Copy" from the appearing menu, which closes automatically. The remaining two context menu options are "Clipboard" (to inspect the current clipboard content) and "Cancel" (to close the menu). Performing press-and-tap on the empty background brings up the clipboard right away. Users can select any of the clipboards items for pasting by touching them. Items are inserted directly at the touch location; the clipboard closes automatically after pasting.

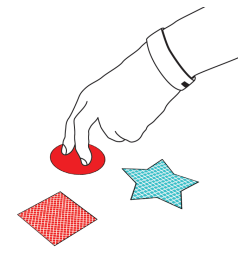

(a)

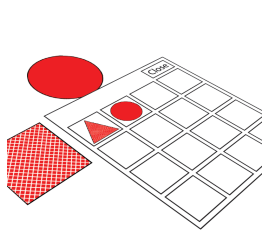

(d)

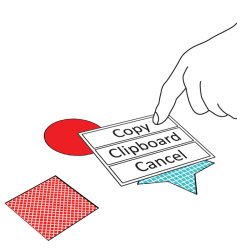

(b)

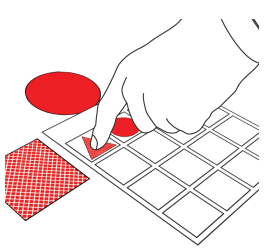

(e)

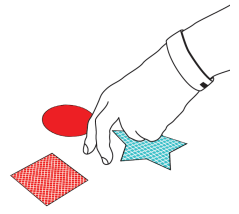

(c)

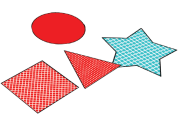

(f)
Figure 2. Copying and pasting with context menu clipboards, based on implicit user identification with IdWristbands. (a) Press-and-tap on item to access menu. (b) Select "Copy" to add selected item to personal clipboard. (c) Press-and-tap on background to access clipboard directly. (d) Current content of clipboard is shown. (e) Touch item to be pasted. (f) Pasted item appears where touched. 


\section{Subarea Clipboards}

Subarea clipboards use the personalization strategy of dynamically associating subregions of the surface to individual users on demand. Any touch input within a personal subarea is associated to the corresponding user. This strategy relies on social protocols to prevent interaction within another user's space. We use HandsDown [22], a biometric user identification technique, to access subarea clipboards. Here, users identify by performing a particular hand gesture: After placing a hand flat onto the surface, with the fingers kept apart, the surface takes a snapshot of the hand and identifies users based on differences in hand shape (e.g., by comparing finger lengths and widths). The area surrounding an identified hand is assigned to the corresponding user as long as the hand stays on the surface. Users interact with their other hand inside this personal area, which automatically closes when removing the identified hand.

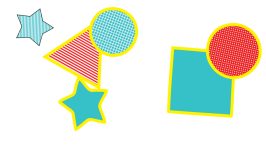

(a)

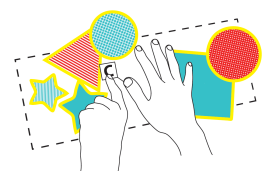

(d)

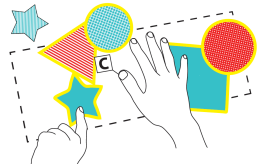

(b)

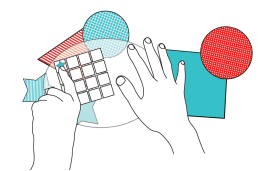

(e)

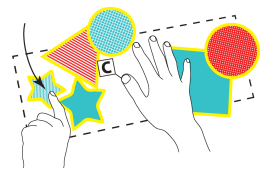

(c)

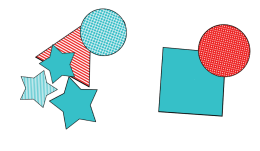

(f)
Figure 3. Copying and pasting with subarea clipboards enabled by HandsDown. (a)-(b) After identification, touch any item inside the clipboard area to copy it. (c) Moving items inside or outside the clipboard area. (d) Access the current clipboard content. (e) Select items for pasting. (f) Pasted items appear where touched.

As illustrated in Figure 3, we appropriate the identification gesture for immediate access to personal clipboards. To copy, users identify next to the items they intend to copy. A dotted rectangle visualizes the personal clipboard area. Any item inside this area is highlighted with a border and can be copied by touching it with the free hand. Users can move items inside the personal area for copying, or move them outside to reduce clutter. The " $C$ " button brings up the current clipboard content, the "X" button switches back to copy mode. To paste, users touch the item to paste, which is inserted right in place. The clipboard stays open while the identification gesture is active, but closes when removing the identified hand.

\section{Handheld Clipboards}

Handheld clipboards use a personalization strategy based on touch input with mobile devices. Similar to using a stylus, users directly touch the surface with the corner of their smart phone. Only touches with the mobile device are identified; finger touches remain anonymous. We used PhoneTouch for touch identification [21]. PhoneTouch is based on simultaneous sensing of touch events on phones (using accelerometers) as well as on the surface (using the integrated camera), and time-based matching of these distributed events. Consequently, each touch performed with a phone is associated to the corresponding device and its user in turn, while phone and finger touches co-exist.

Phone touches provide another modality in addition to finger touches, which we use for direct access to copy-and-paste. We permanently show the clipboard content on the phones' screen without occupying surface space. As illustrated in Figure 4, users touch an item with the phone to copy it, using either of the phone's two top corners. The copied item instantly appears in the clipboard shown on the phone. To paste, users first select items on the phone screen and then perform a phone touch on the surface; pasted items appear where touched. Moving, resizing, or rotating items on the surface (e.g., to bring them closer or align them) requires finger input; phone touches are reserved for clipboard interactions.

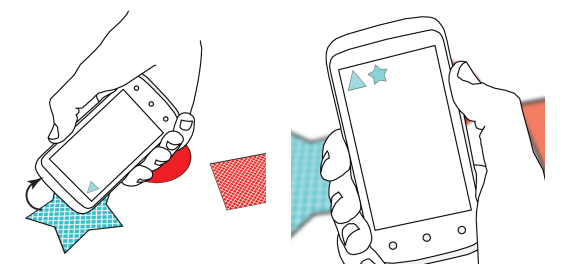

(a)

(b)

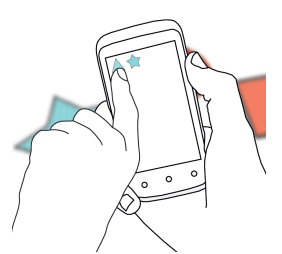

(c)

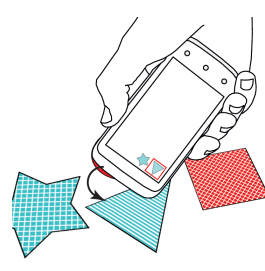

(d)
Figure 4. Copying and pasting with handheld clipboards enabled by PhoneTouch. (a) Touch an item with the phone for copying. (b) The clipboard content is permanently shown on the phone. (c) Select items for pasting on the phone screen. (d) Touch with the phone to paste items back to the surface.

\section{STUDY DESIGN}

The goal of the following user study is to demonstrate the effectiveness of the proposed systems, and to explore how users interact with them. We are particularly interested in how the traditional clipboard concept carries over from single-user devices to multi-user surface computing. At the same time, we compare different personalization strategies in order to gain insights into their impact on interaction characterstics.

To this end, we recruited nine pairs of participants from our local campus through posters and mailing lists (eight female; aged 19 to 29 years, $M=22.35, S D=3$ ). Participants of five groups $(56 \%)$ were acquaintances, the others did not know each other beforehand; two groups were mixed-gender. All but one participant were right-handed. Most participants reported a "high" experience (i.e., 4 on a 5-point rating scale) with computers in general and touch interfaces in particular. Only three participants had used a large multi-touch surface before, but many were familiar with direct touch smart phones $(67 \%)$ or tablets $(22 \%)$. Each participant received $£ 8$ for their time. 


\section{Apparatus}

We implemented all three clipboards with off-the-shelf surface hardware, using the Samsung SUR40 device (Microsoft PixelSense). The SUR40 has a height of $73 \mathrm{~cm}$ and a surface diagonal of $120 \mathrm{~cm}$ at a resolution of 1920 pixel $\times 1080$ pixel. Only the context menu clipboard, based on IdWristband, requires users to wear custom hardware for identification. The subarea clipboad is self-contained in the surface, and the handheld clipboard integrates off-the-shelf mobile phones for user identification (i.e., iPhone $3 \mathrm{GS}$ equipped with protective rubber bumpers). We developed the study task application in C\# using Microsoft's .NET framework, Windows Presentation Framework (WPF), and the Surface 2.0 SDK. In each condition, participants sat at the longer table sides opposite each other (see Figure 5).

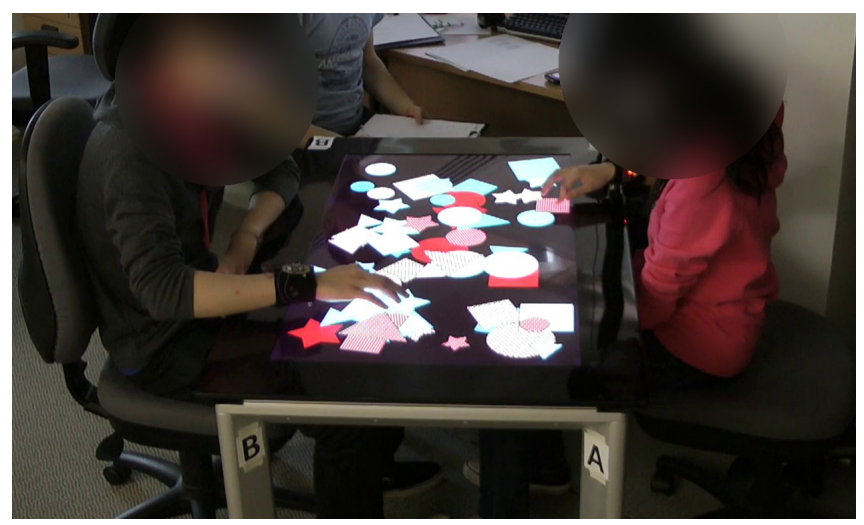

Figure 5. Participants during the study (using context menu clipboards)

\section{Task}

The study consists of a copy and a paste sub-task, carried out one after the other. We asked participants to first copy and then paste a series of geometric shapes. These items differed in three features (see Figure 6(a)): color $(\times 2)$, pattern $(\times 4)$, and shape $(\times 4)$. We presented each unique combination of features twice, resulting in 64 items available for copying. Before starting, we asked participants to choose a color.

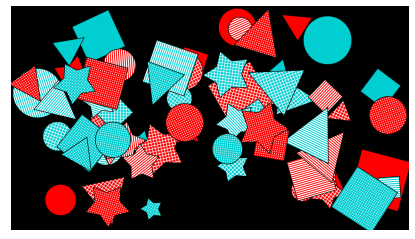

(a)

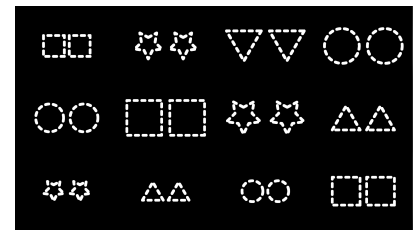

(b)
Figure 6. Copy and paste tasks: (a) First, participants copied 16 items of the same color but different in pattern and shape. (b) In the second step, 12 target locations indicated where to paste the just copied items.

\section{Copy}

During the copy task, participants had to search for and copy multiple items into their personal clipboards. We instructed them to find all 16 unique combinations of shape and pattern of their chosen color. As shown in Figure 6(a), we randomly arranged items in the beginning. Participants could move, rotate, and resize any item using typical multi-touch interactions.
Items flashed as confirmation after copying them. We did not allow copying of duplicate items (i.e., same color, pattern, and shape) to prevent participants from rushing through the task by copying random items. Any attempt to copy duplicates was visualized with a shaking animation. To remove an item from the clipboard, participants had to tap and hold it (i.e., on the surface in case of context menu and subarea clipboards, and on the phone in case of handheld clipboards).

\section{Paste}

Once participants had copied the requested items, we switched to a second screen. Here, participants had to paste a selection of their copied items, matching the 12 target locations shown in Figure 6(b). For each target location, participants had to select and arrange two items that differed in both color and pattern, but had the same shape, as indicated by the dotted outlines. This was to foster collaboration: Each participant had to contribute one item to every target location. In addition, participants had to coordinate to ensure they selected different patterns.

\section{Conditions}

We used a within-subject repeated-measures design with the independent variable system (context menu, subarea, or handheld clipboard). Participants performed copy and paste tasks for all three systems, sitting opposite each other (see Figure 5). The presentation of systems was counter balanced.

\section{Procedure}

Participants first signed research consent forms. Then, the experimenter explained the copy and paste tasks. Before using each system, the experimenter demonstrated the identification technique and how to perform copy-and-paste interactions; participants tested the system until they felt comfortable using it. In case of subarea clipboards, we asked participants to register for HandsDown beforehand by placing their hands repeatedly on the surface. The copy task was completed once both participants had collected 16 different items of their color. The paste task was completed once both participants had pasted and arranged items matching the indicated target locations. Task completion was not automatically registered but determined by the experimenter.

Throughout the task, we observed participants and took notes. We video-taped all sessions for detailed post-hoc analysis using an open coding approach [31] and ChronoViz to facilitate annotations [34]. Our field notes provided a starting point for initial coding categories. A detailed system interaction log complements this video analysis.

After completing the tasks with each system, we asked participants to state their agreement with eight items selected from the IBM Computer Usability Satisfaction questionnaire ${ }^{1}$ on a seven-point Likert scale ranging from "strongly agree" to "strongly disagree". Further, using three items from the NASA Task Load Index, we asked participants to rate the amount of mental demand required to fulfil the task as well as their frustration level, and to give a self-assessment of their performance. After completing all conditions, we asked participants

\footnotetext{
${ }^{1}$ Statements 2, 4, 5, 6, 7, 9, 17, and 19 of the Post-Study System Usability Questionnaire were selected as applicable here.
} 
to rank systems according to several criteria, including general preference as well as their perception regarding execution time, efficiency, enjoyment, learnability, and responsiveness. Finally, we conducted an open-ended interview to gain additional insights into particular interaction patterns we had observed.

\section{RESULTS}

Participants readily understood the study tasks, and were able to successfully complete tasks independent of the system. They could instantly identify to access their clipboards when and where required. We did not observe any fundamental difficulties in using copy-and-paste with any of the tested systems. In the following analysis, we first summarize and analyze the collected user feedback and then report on results from our video and system log analysis.

\section{User Feedback}

The quantitative user feedback provided a balanced picture of the three systems and did not reveal differences of substance. We did not find significant differences amongst the responses to the 11 selected items from the usability and task load questionnaire (using Friedman's ANOVA). Neither did the system ranking reveal clear preferences for one of the systems, as shown in Figure 7.

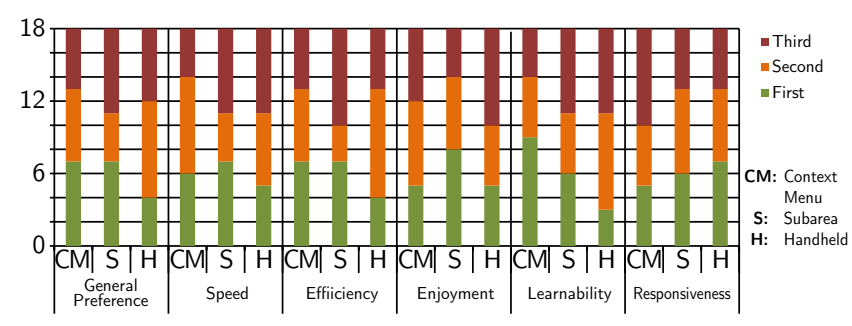

Figure 7. Ranking results show the number of participants who picked a particular system as first (green), second (orange), or third choice (red); they do not reveal clear preferences.

The qualitative user feedback, on the other hand, provided more insights into differences between the three systems. Using context menu clipboards, none of the participants felt impeded by the wristbands, and nine participants explicitly mentioned that wearing wristbands was not cumbersome or uncomfortable. Some compared the experience to wearing regular accessories, such as wrist watches, or perceived wristbands as being "invisible" (participant 9A). We received positive comments from nine participants about the implicitness and subtleness of identified interaction enabled by IdWristbands. Three emphasized the consistency with regular touch screen interaction, as familiar touch styles could be used without having to adapt for identification (8A: "it's like normal finger motion").

Using subarea clipboards, seven participants appreciated the fact that no user instrumentation or additional devices were required for identification. None of the participants brought up privacy issues with respect to using biometrics. Two participants, however, raised concerns about the available surface space; one of them found the overlapping of lenses "irritating" (3B). Another participant felt more comfortable using subarea compared to context menu clipboards as it was possible to put the "hand down to rest" to open up an identification area, rather than having to "keeping hands up", which was considered tiring (6A). Five other participants would have preferred having both hands available.

Using handheld clipboards, four participants appreciated the mobile phone as a familiar and readily available device. Two participants actually preferred interacting with the phone on the surface compared to using fingers, while another one commented that replacing finger touch entirely by phone touch was not desirable. The same participant felt that using the phone for touch interaction was not intuitive (6B). None of the participants raised concerns with respect to potential damage to the phone or the surface. One participant saw the phone as dedicated tool and highlighted that there were "no gestures or sequences to remember" to copy and paste (3A). The additionally available phone screen was welcomed by 10 participants, as it allowed them to permanently see and quickly inspect what had already been copied. Five participants gave positive feedback about the instantaneous transfer of data between devices (3B: "transferring objects to the telephone was very cool"). None of the participants commented about having to alternate hands for interaction on the surface or on the phone.

\section{Completion Times}

Figure 8 summarizes completion times for each system and subtask. The system had a significant effect on copy times $\left(\chi^{2}(2)=12.6, p<.05\right.$; using Friedman's ANOVA due to non-normal data distribution), but not on paste times. We used Wilcoxon tests to follow up on these findings and applied a Bonferroni correction, hence all effects are reported at a .017 significance level. The only significant difference emerged for copying with handheld clipboards, which was significantly faster compared to context menu $(T=0, p<.017, r=-.62)$ and subarea clipboards $(T=1, p<.017, r=-.6)$.

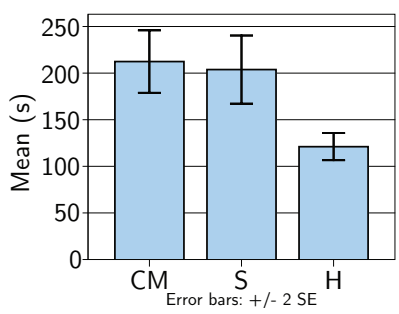

(a) Copy

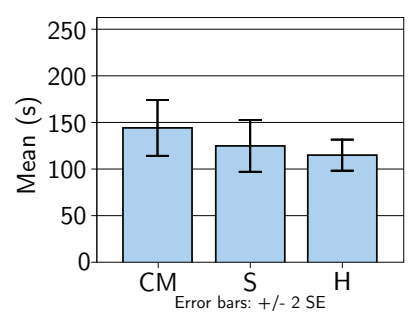

(b) Paste
Figure 8. Mean completion times of copy and paste tasks are similar for all systems; only copying with handheld clipboards was significantly faster than copying with the other systems (CM: Context Menu, S: Subarea, H: Handheld).

\section{Surface Utilization}

To explore possible impacts of the different personalization strategies on interaction characteristics, we analyzed the spatial distribution of copy and paste activities (i.e., activities that required the user to be known) with respect to the following two indicators: vicinity-i.e., percentage of copy or paste activities within the surface half closest to a participant—and coverage - i.e., percentage of covered surface area based on 


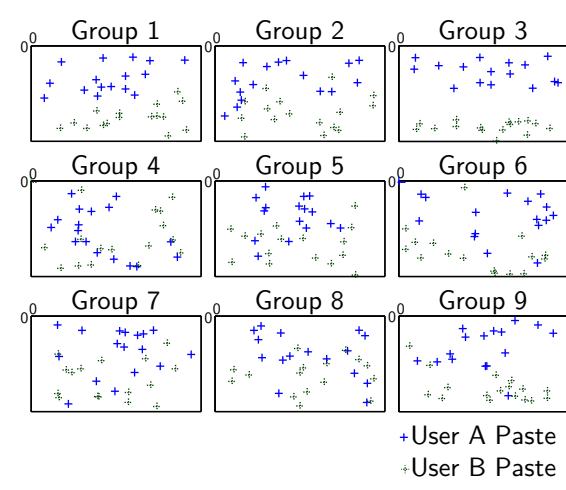

(a) Context menu
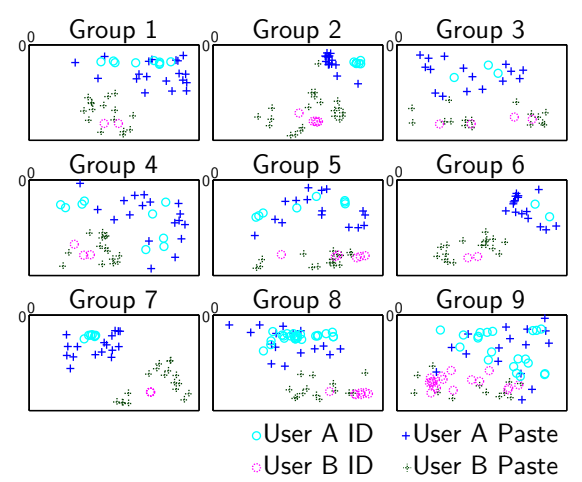

(b) Subarea
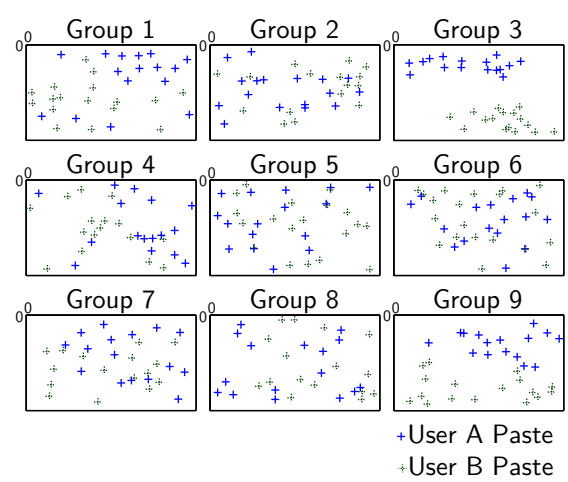

(c) Handheld

Figure 9. Locations at which participants copied items. Crosses indicate copied items, circles HandsDown identification gestures. Participants spread out the most using (c) handheld clipboards followed by (a) context menu clipboards; using (b) subarea clipboards, items were moved before copying.

the convex hull (the smallest convex set) of copy or paste locations. Figure 10 summarizes space utilization for the three systems.

\section{Copy}

Figure 9 plots locations of all copy activities for each system and participant group. Using context menu clipboards, six participants (groups 4, 7, and 8) spread out more than average and performed at least $25 \%$ of copy activities within the opposite table half. In contrast, participants of group 3, who had sorted items by color prior to copying, stayed within their respective halves throughout.

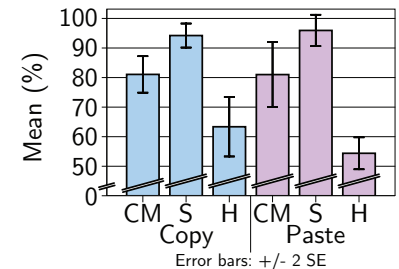

(a) Copy and paste vicinity

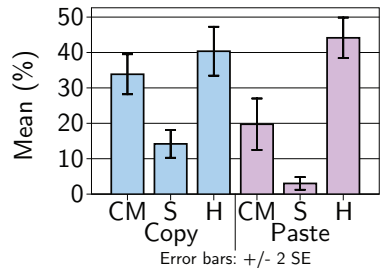

(b) Copy and paste coverage
Figure 10. Surface utilization was most spread out for handheld clipboards, followed by context menu and subarea clipboards, independent of copy or paste (CM: Context Menu, S: Subarea, H: Handheld).

Unlike context menu clipboards, subarea clipboards required an explicit identification gesture for access. We found that participants accessed their clipboard 7.94 times on average $(S D=6.93)$. All but two participants (4A and 9A) did so within their half of the table. In general, identification areas were respected. Five participants, however, occasionally dragged items directly out of the other participant's area. This was generally accepted except for once when the disadvantaged participant removed his hand from the surface. We observed two prevalent approaches to copying with subarea clipboards: Half of the participants primarily dragged items towards the identified hand, keeping its position mostly unchanged, while the other half frequently varied the identification location, bringing the hand closer to items in question.

Using handheld clipboards, all but two participants had the phone screen generally facing them. We also observed that participants frequently had a closer look at their phone to inspect copied items. Compared to the other two systems, participants used more of the available surface area.

\section{Paste}

Figure 11 plots locations of all paste activities for each system and participant group, revealing similar distributions as for copying. Using context menu clipboards, half of the participants pasted most items close to the designated target locations, while the remaining half accessed clipboards at seemingly arbitrary positions, arranging items only after inserting them. Some following the latter approach, however, decided on a rough location before accessing the clipboard.

Using subarea clipboards, participants placed their hand for identification 4.56 times on average $(S D=3.31)$. Participants did not vary identification locations much and pasted items grouped together independent of target locations. They performed all HandsDown gestures within their own table half. Most participants ( $72 \%$ ) pasted one or multiple items at a time to then arrange them before proceeding, while three participants pasted all items at once to arrange them in a separate step.

Having the handheld clipboard on a separate screen allowed participants to inspect both remaining items on the phone and targets to fill on the surface at the same time, without occluding the shared work space. Two participants pasted most items in batches to arrange them together. The majority, however, interleaved paste and (finger-based) arrange interactions to directly insert items at the intended target locations, even if close to the other participant, resulting in a clearly visible pattern (see Figure 11(c)).

\section{Analysis}

Copy-and-paste interactions typically took place closer to the corresponding participant for subarea than for handheld clipboards; context menu clipboards lie in between. Likewise, copy-and-paste interactions of subarea clipboards covered the smallest area, followed by context menu and then handheld clipboards. Applying a Friedman ANOVA, we found that all four measures were significantly affected by the system: copy vicinity $\left(\chi^{2}(2)=20.49, p<.05\right)$, copy coverage $\left(\chi^{2}(2)=\right.$ 


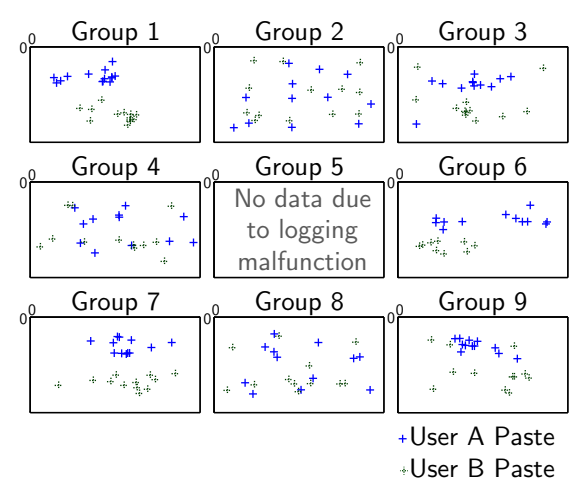

(a) Context menu
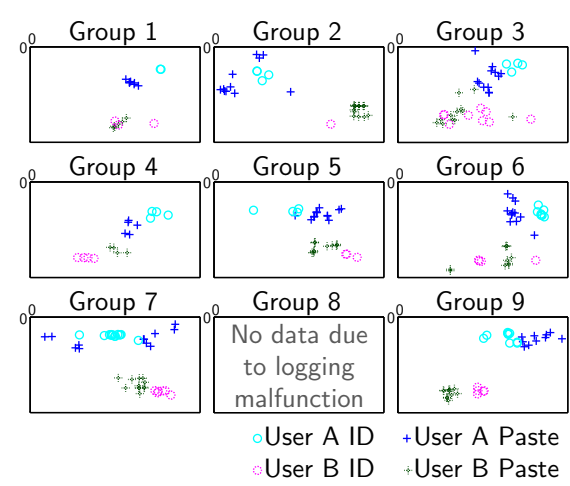

(b) Subarea

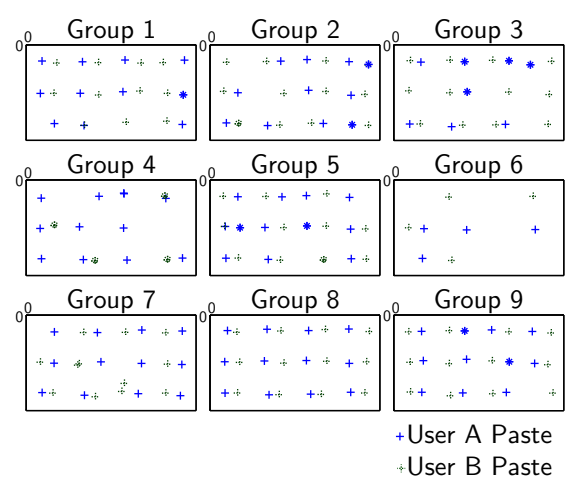

(c) Handheld

Figure 11. Locations at which participants pasted items. Crosses indicate copied items, circles HandsDown identification gestures. The target location pattern is clearly visible for pasting with (c) handheld clipboards, while participants pasted in clusters using (b) subarea clipboards.

$16.44, p<.05)$, paste vicinity $\left(\chi^{2}(2)=17.33, p<.05\right)$, and paste coverage $\left(\chi^{2}(2)=22.43, p<.05\right)$. Wilcoxon posthoc tests revealed (Bonferroni-corrected significance level of .017) that there were no significant differences between handheld and context menu clipboards for copy coverage and between subarea and context menu clipboards for paste vicinity; all other pairwise tests showed significant effects. Applying Mann-Whitney tests on coverage and vicinity results, we found that participants who knew each other beforehand did not differ from those who were not acquainted with respect to their surface space utilization.

\section{Handedness}

Using context menu clipboards, all but one participant choose to wear wristbands on the dominant hand. Although the other hand was available for (anonymous) touch input (e.g., to move or resize items), all but two participants limited themselves to one-handed input.

Using subarea clipboards, participants could perform HandsDown gestures with either of their hands. Including both copy and paste tasks, we observed that 12 participants $(67 \%)$ consistently stayed with the same hand for identification (seven used the dominant and five the non-dominant hand), while the remaining six alternated at least once.

Using handheld clipboards, all but one participant set out using the dominant hand to hold the phone and copy items. While copying, six participants switched hands, for example to delete an item they had copied by mistake, to better reach the opposite table side, or to use the hand for a finger-based interaction on the surface. Participants seldom used finger touch input on the surface when copying. In fact, six participants (33\%) exclusively relied on phone touches. Even if items were partially occluded, participants could copy them directly by touching visible parts with the phone corner.

Unlike copying, pasting required both phone and finger interaction to align items with target locations on the surface. Participants also had to select items to paste on the phone screen. We observed various approaches with regard to hand preferences and alternating hands for the different interaction types. Eight participants $(33 \%)$ kept the phone primarily in one hand. The remaining 10 participants $(67 \%)$ frequently alternated hands as they saw fit. To illustrate a possible interaction flow, we describe the approach consistently followed by two participants (see Figure 12): Keeping the phone in their non-dominant hand, they selected items to paste using fingers of the dominant hand (a). Before touching the surface with the phone, they turned it over to the dominant hand (b), pasted items (c), and handed it immediately back to the nondominant hand (d) for arranging the just pasted item using finger input on the surface (e). This sequence was repeated for the remaining items (f). Approaches varied greatly amongst participants, however. For example, some switched hands only occasionally for finger interaction with the dominant hand on the surface, while otherwise performing such input with the non-dominant hand.

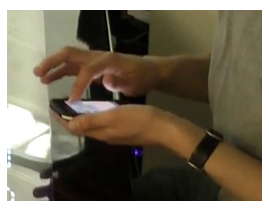

(a)

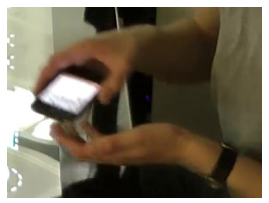

(d)

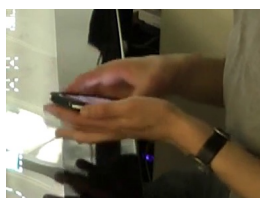

(b)

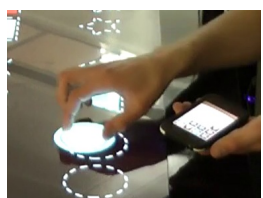

(e)

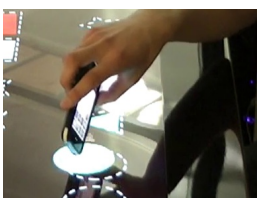

(c)

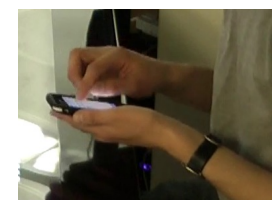

(f)
Figure 12. Interleaving finger and phone touches using handheld clipboards: (a) Selecting an item on the phone before (b) changing hands (c) to paste it on the surface. (d) Changing hands (e) to arrange the item on the surface. (f) Continuing with the next item.

\section{DISCUSSION}

Participants immediately grasped the idea of personal clipboards and were able to efficiently use the three systems with only minimal training. We showed that user identification allows for directly transferring the familiar clipboard concept from single-user devices to multi-user surface computing. It 
was apparently taken for granted that each participant could copy to, and paste from, their own clipboard without interference. This implies that participants seemed to expect interactions to be associated to individual users.

Personal clipboards make advantages of traditional clipboards available on shared surfaces. Source and target of copy-andpaste actions do not have to be reachable or even visible at the same time. Therefore, clipboards provide a convenient alternative to drag-and-drop over long distances, or when pasting does not immediately follow copying. This enables users to perform other activities in between. Unlike the shared surface, personal clipboards provide a space exclusive to their owners to collect content without interference.

Despite their different approaches, all tested personalization strategies successfully enabled individual copy-and-paste. In general, the strategy did not influence task completion times significantly. The only exception was the faster copying with handheld clipboards. In contrast to the other systems, handheld clipboards did not require selecting the copy option before each interaction (this could be done once on the phone), resulting in a simple pointing interaction for copying. While no general preference for any of the systems emerged, the underlying identification strategies had a clear impact on interaction characteristics.

\section{Limitations}

Regardless of its abstract nature, we are confident that our study task reveals interaction qualities that also apply to typical surface applications. In fact, we chose an abstract task so that the study results are independent of specific applications. The task is designed to feature the kind of copy-and-paste interactions that occur in realistic usage scenarios. In particular, participants had to sift through and consider multiple items before copying, leading to a frequent interleaving of browsing and copying interactions. Similarly, participants interleaved pasting and aligning items, while working closely together to achieve the set goals, resembling a typical collaborative task.

While the chosen setup of two users sitting on opposite table sides is common, we did not study other settings (e.g., side by side or additional users). However, we expect that our findings still apply, as personal clipboards are accessible from any surface location. Nevertheless, the closer users sit together, or the more users share the same table, the more likely are interferences - just as with regular surface interactions. Application designers therefore need to carefully choose size and orientation of context menu and subarea clipboards.

\section{Implications}

Users typically organize their workspaces, form different territories, and coordinate collaboration. Unlike the territories identified by Scott et al. [26], personal clipboards are not a permanent part of the workspace, but represent a complementary concept. Therefore, copy-and-paste needs to be accessible independent of surface regions. This was best supported by handheld clipboards, which encouraged participants to widely spread out their copy-and-paste activities, thereby covering the entire surface. Here, users could make selections in advance on the phone; interaction on the surface then narrowed down to a single touch. In contrast, both context menu and subarea clipboards required additional selection steps on the surface.

Surface interaction benefits from the freedom of using multiple fingers and hands for expressive input. Subarea and handheld personalization strategies require supplementary interactions and limit how hands can be used for input. Our analysis of handedness revealed, however, that both strategies were seamlessly integrated into the interaction flow. Moreover, the dedicated HandsDown identification gesture can be overloaded with additional functions (e.g., invoking a clipboard). Similarly, using the phone for touches is comparable to using a configurable tool, like a Swiss army knife: Users can pre-select options (using the built-in GUI) to be applied to the next surface touch (e.g., selecting the item to paste). In comparison to implicitly associating finger touches, assigning regions and using handheld devices allows users to control the scope of identified input; remaining anonymous is as simple as performing a regular finger touch.

Copy-and-paste with subarea clipboards has the highest costs with respect to identification time and surface space utilization. Further, it is difficult to perform HandsDown gestures far from where users are located. Therefore, users need to be able to easily move items they wish to copy into reach. Alternatively, lenses can stay open when lifting off the hand. They can then be moved around and eventually be closed manually (i.e., after copy or paste interactions are completed). As a third of our participants alternatively used both their left and right hand to access clipboards, application designers need to ensure that lens shapes and layouts adapt accordingly.

The separate screen of handheld clipboards was frequently used by participants. Therefore, information presented on the phone needs to be also legible when interacting on the surface (e.g., layout, size, or orientation of content can be changed during copy-and-paste interactions). Furthermore, participants often used phones in lieu of fingers for direct pointing. As pointing with phones results in a larger occlusion of the surface, applications may need to increase the minimum size of user interface elements.

\section{CONCLUSION}

We have introduced personal clipboards for shared surfaces and demonstrated that this familiar concept directly transfers to individual copy-and-paste operations in multi-user environments, once users can be identified. Based on three system implementation, our study showed that personal clipboards can be realized with different personalization strategies. We demonstrated that implicitly identifying any finger touch is not required for fluid interaction. Therefore, choosing a personalization strategy may be guided by practical considerations (e.g., available hardware) or required functionalities (e.g., presenting content on separate devices). We leave the exploration of how further GUI concepts that are familiar from single-user devices can be transferred to shared surfaces capable of user identification for future work.

\section{REFERENCES}

1. Bederson, B. B. Interfaces for staying in the flow. Ubiquity 2004 (September 2004), 1-1. 
2. Block, F., Gellersen, H., and Villar, N. Touch-Display Keyboards: Transforming keyboards into interactive surfaces. In Proc. CHI (2010), 1145-1154.

3. Block, F., Villar, N., and Gellersen, H. A malleable physical interface for copying, pasting, and organizing digital clips. In Proc. TEI (2008), 117-120.

4. Chapuis, O., and Roussel, N. Copy-and-paste between overlapping windows. In Proc. CHI (2007), 201-210.

5. Deutsch, L. P., and Lampson, B. W. An online editor. Commun. ACM 10, 12 (Dec. 1967), 793-799.

6. Dietz, P. H., and Leigh, D. DiamondTouch: A multi-user touch technology. In Proc. UIST (2001), 219-226.

7. Dix, A., Catarci, T., Habegger, B., loannidis, Y., Kamaruddin, A., Katifori, A., Lepouras, G., Poggi, A., and Ramduny-Ellis, D. Intelligent context-sensitive interactions on desktop and the web. In Proc. AVI (2006), 23-27.

8. Everitt, K., Shen, C., Ryall, K., and Forlines, C. Modal spaces: spatial multiplexing to mediate direct-touch input on large displays. In CHI Ext. Abstracts (2005), 1359-1362.

9. Hartmann, B., Ringel Morris, M., and Cassanego, A. Reducing clutter on tabletop groupware systems with tangible drawers. Poster UbiComp, 2006.

10. Hutama, W., Song, P., Chi-Wing, F., and Goh, W. B. Distinguishing multiple smart-phone interactions on a multi-touch wall display using tilt correlation. In Proc. CHI (2011), 3315-3318.

11. Kjeldskov, J., Skov, M., Als, B., and Høegh, R. Is it worth the hassle? exploring the added value of evaluating the usability of context-aware mobile systems in the field. In Proc. MobileHCI (2004), 61-73.

12. Meyer, T., and Schmidt, D. IdWristbands: IR-based user identification on multi-touch surfaces. Poster ITS, 2010.

13. Microsoft. Using touch gestures. 27 April 2012. http://windows . microsoft . com/en-US/windows 7/ Using-touch-gesture.

14. Miller, R. C., and Myers, B. A. Synchronizing clipboards of multiple computers. In Proc. UIST (1999), 65-66.

15. Myers, B. A., Miller, R. C., Bostwick, B., and Evankovich, C. Extending the windows desktop interface with connected handheld computers. In Proc. USENIX Win. Sys., vol. 4 (2000), 8.

16. Pinelle, D., Stach, T., and Gutwin, C. TableTrays: Temporary, reconfigurable work surfaces for tabletop groupware. In Proc. TableTop (2008), 41-48.

17. Rekimoto, J. Pick-and-Drop: A direct manipulation technique for multiple computer environments. In Proc. UIST (1997), 31-39.

18. Richter, S., Holz, C., and Baudisch, P. Bootstrapper: Recognizing tabletop users by their shoes. In Proc. CHI (2012), 1249-1252.
19. Rofouei, M., Wilson, A., Brush, A., and Tansley, S. Your phone or mine?: fusing body, touch and device sensing for multi-user device-display interaction. In Proc. CHI (2012), 1915-1918.

20. Roth, V., Schmidt, P., and Güldenring, B. The IR Ring: authenticating users' touches on a multi-touch display. In Proc. UIST (2010), 259-262.

21. Schmidt, D., Chehimi, F., Rukzio, E., and Gellersen, H. PhoneTouch: A technique for direct phone interaction on surfaces. In Proc. UIST (2010), 13-16.

22. Schmidt, D., Chong, M., and Gellersen, H. HandsDown: Hand-contour-based user identification for interactive surfaces. In Proc. NordiCHI (2010), 432-441.

23. Schmidt, D., Chong, M., and Gellersen, H. IdLenses: Dynamic personal areas on shared surfaces. In Proc. ITS (2010), 131-134.

24. Schmidt, D., Seifert, J., Rukzio, E., and Gellersen, H. A cross-device interaction style for mobiles and surfaces. In Proc. DIS (2012), 318-327.

25. Scott, S., Carpendale, S., and Habelski, S. Storage bins: Mobile storage for collaborative tabletop displays. IEEE Comp. Graph. and App. 25 (2005), 58-65.

26. Scott, S., Carpendale, S., and Inkpen, K. Territoriality in collaborative tabletop workspaces. In Proc. CSCW (2004), 294-303.

27. Shen, C., Ryall, K., Forlines, C., Esenther, A., Vernier, F., Everitt, K., Wu, M., Wigdor, D., Morris, M., Hancock, M., and Tse, E. Informing the design of direct-touch tabletops. IEEE Comp. Graph. and App. 26 (2006), 36-46.

28. Smith, D. C., Irby, C., Kimball, R., Verplank, B., and Harslem, E. Designing the star user interface. Byte 7, 2 (1982), 242-282.

29. Stolee, K., Elbaum, S., and Rothermel, G. Revealing the copy and paste habits of end users. In IEEE Symp. on VL/HCC (September 2009), 59 -66.

30. Stylos, J., Myers, B. A., and Faulring, A. Citrine: providing intelligent copy-and-paste. In Proc. UIST (2004), 185-188.

31. Tang, A., Tory, M., Po, B., Neumann, P., and Carpendale, S. Collaborative coupling over tabletop displays. In Proc. CHI (2006), 1181-1190.

32. Tesler, L. A personal history of modeless text editing and cut/copy-paste. interactions 19, 4 (July 2012), 70-75.

33. Wallace, G., Biddle, R., and Tempero, E. Smarter cut-and-paste for programming text editors. In Proc. AUIC (2001), 56-63.

34. Weibel, N., Fouse, A., Hutchins, E., and Hoolan, J. Supporting an integrated paper-digital workflow for observational research. In Proc. IUI (2011), 257-266.

35. Wu, M., Shen, C., Ryall, K., Forlines, C., and Balakrishnan, R. Gesture registration, relaxation, and reuse for multi-point direct-touch surfaces. In Proc. TableTop (2006), 185-192. 